\title{
Time To Death Among HIV-Infected Under-Five Children After Initiation Of Anti-Retroviral Therapy And Its Predictors In Oromiya Liyu Zone, Amhara Region, Ethiopia: A Retrospective Chohort
}

Weldemariam Sintayehu Arega

Bati District Health Office

Bereka Tefera Marie ( $\square$ ttmarie52@gmail.com )

Kotebe Metropolitan University

Bitewa Yibelu Bazezew

Debre Markos University

\section{Research Article}

Keywords: Antiretroviral Therapy, Under-Five Children, Death, Northeast, Ethiopia.

Posted Date: February 24th, 2021

DOl: https://doi.org/10.21203/rs.3.rs-234377/v1

License: (c) (1) This work is licensed under a Creative Commons Attribution 4.0 International License.

Read Full License 
1 TIME TO DEATH AMONG HIV-INFECTED UNDER-FIVE CHILDREN AFTER 2 INITIATION OF ANTI-RETROVIRAL THERAPY AND ITS PREDICTORS IN OROMIYA 3 LIYU ZONE, AMHARA REGION, ETHIOPIA: A RETROSPECTIVE CHOHORT

4 Weldemariam Sintayehu Arega ${ }^{1}$, Bereka Tefera Marie ${ }^{2}$, Bitewa Yibelu Bazezew ${ }^{3}$

5 Authors Affiliation

$6 \quad{ }^{1}$ Public Health Emergency Management Officer, Bati District health office, Ethiopia

$7 \quad{ }^{2}$ Lecturer, Midwifery Department, Kotebe Metropolitan University, Ethiopia

$8 \quad{ }^{3}$ Lecturer, Midwifery Department, Debremarkos University, Ethiopia

9

10 Corresponding author: Bereka Tefera Marie: ttmarie52@gmail.com

11

12 


\section{ABSTRACT}

Background: Human Immunodeficiency Virus (HIV) is infection which mainly attacks immune system of an individual. Its disease progress is rapid in children and if treatment is not initiated nearly half of infected children will die by the second year of infection. In Ethiopia, nearly twenty four percent of HIV related death is happen to under-five children; however studies done in this specific age group are limited are with poor evidence of predictors.

Objectives: To determine time to death and identify predictors of death in HIV infected underfive children on antiretroviral therapy in Amhara regional state, Oromia 'liyu' zone, Northeast Ethiopia, from 2014 to 2019.

Methods: Institution based retrospective follow up study was conducted in 376 under-five HIVinfected children on antiretroviral therapy from January 2014 to December 2019 in health institutions in Oromia Liyu Zone, Amhara region, Ethiopia. Multivariable Cox-proportional hazard regression model was used to identify independent predictors of Mortality in HIVinfected under-five children on antiretroviral therapy.

27 Results: At the end of follow up, 285 (80.28\%) of HIV-infected children were alive, 39 $(10.99 \%)$ were lost to follow up, 11 (3.94\%) were transferred out and 21 (5.59\%) were reported dead due to HIV/AIDS. The cumulative survival probabilities of children after 3, 6, 12, 24 and 36 months were $0.99,0.98,0.97,0.89$ and 0.87 respectively. The overall mean time to death was 19.7 months $(95 \% \mathrm{CI}=18.74-20.67)$ with incidence of 5.9 deaths per 100 child-months (95\%CI: 3.89-9.09). Children with severe malnutrition at baseline (AHR=4.9; $95 \mathrm{CI}$ : 1.04, 23.50), advanced WHO clinical stage at enrolment (AHR=3.9; CI: 1.37, 10.88), poor adherence to ART $(\mathrm{AHR}=6.56 ; \mathrm{CI}: 3.33,10.14)$ and with no history of Isoniazide prophylaxis were significantly associated to higher mortality events (AHR=3.6; CI: 1.24, 10.18).

Conclusion: Death of HIV-infected under-five children on ART is high within the first one year after enrolment. The risk of death increased if the child was malnourished at begging of treatment, had poor ART adherence, with advanced WHO clinical stages and lack of Isoniazide prophylaxis during their age of infancy.

Key words: Antiretroviral Therapy, Under-Five Children, Death, Northeast, Ethiopia. 
42 HIV is Human Immunodeficiency Virus (HIV) infection which mainly attacks immune system 43 of an individual. It rapidly progresses in under-five children, if not put on a treatment nearly half 44 of them would be dead by the second year of infection $(\underline{1}, \underline{2})$. The natural history and clinical 45 manifestations of HIV differs between children and adults, with more rapid disease progression 46 in infancy and a predilection for recurrent, common viral and bacterial infections over 47 opportunistic infections. The more rapid progression is attributed due to an immature immune 48 system, and the effect of viral replication and inflammation on somatic and neuro-developmental growth. The opportunistic infections that result from immune deficiency in under-five children with HIV are often primary, not reactivation disease, thereby resulting in greater morbidity. Despite these challenges, the great strides made in the treatment of children with HIV have positively impacted disease progression and immune capacity, which continue to improve survival rates and quality of life (1-3).

54 The majority of under-five children living with HIV are infected via mother-to-child transmission (MTCT), during pregnancy, childbirth or breastfeeding. This can be declined as long as expectant mothers have access to preventing mother-to-child transmission (PMTCT) 57 services during pregnancy, delivery and breastfeeding. More over WHO recommends that infants exposed to HIV to be tested at the first postnatal visit, usually when they reach four to six weeks of age or at the earliest opportunity thereafter, and infants who are infected start treatment immediately. However, identifying those infants by using the common antibody HIV test is a challenge due to the presence of maternal HIV antibodies, which may persist for as long as 18 months in a child's bloodstream (1-4).

63 Highly Active Antiretroviral Treatment (HAART) consistently improved survival of under-five children infected with the virus and contributed to prevent new infections. The risk of a mother living with HIV passing the virus to her child can be reduced to $5 \%$ or less if she has access to 66 effective antiretroviral therapy during pregnancy, delivery and breastfeeding $(\underline{1}, \underline{3})$. Without any 67 intervention for prevention of mother-to-child HIV transmission (PMTCT), 15-45 percent of HIV-positive women will transmit HIV to their babies, depending on the length of time an infant 69 is breastfed $(\underline{1}, \underline{3-5})$. 
Worldwide under-five children death due to HIV/AIDS accounted for $1.4 \%$ in 2015 and over

71 120,000 children died due to AIDS-related illnesses in 2016, this equates to 328 deaths every

72 day. In fact, children aged 0-4 years living with HIV are more likely to die than any people

73 living with HIV of any other age; this is despite a $62 \%$ reduction in AIDS-related deaths and

$7470 \%$ decline in the number of new HIV infections among this age group since 2000, as a result

75 of scaled-up HIV prevention services $(\underline{2}, \underline{6})$. Despite this significant progress, the number of

76 under-five children becoming newly infected and dead with HIV remains unacceptably high, for

77 instance about 150000 children became infected with HIV in 2015, down from 490000 in 2000

$78(\underline{2}, \underline{4}, \underline{7}, \underline{8})$.

79 In Ethiopia estimated 722,248 people are living with HIV, current prevalence is $1.16 \%$, with 80 children accounting for around ten percent. According to 2017 report of CDC, HIV/AIDS is the $817^{\text {th }}$ cause of death in Ethiopia affecting children at high rate (4). Moreover, twenty four percent 82 of all AIDS-related deaths are among under-five children, more than thirty percent of people with new infections are children, and ART coverage for children is around 34\%, below the national target of having 85 percent of children living with HIV on ART by 2020. In 2018 PMTCT coverage, which is assumed to be major preventive factor for under-five child deaths, was still in between 63 to 93 percent, which needs high effort. Coverage of early infant diagnosis of HIV is estimated at around seventy percent and in 2019 from all causes of under-five mortality HIV/AIDS accounts for $2 \%(\underline{1}, \underline{3}, \underline{9})$.

Although visible efforts aimed at improving HIV infected child survival have driven large reductions in mortality levels among children under- five years of age, presence of persistent and intolerably high numbers of child and young adolescent deaths mean more work remains to be done to address the specific survival needs of children and young adolescents in Ethiopia (10, 11). So this study is aimed at addressing survival status and magnitude of deaths in HIV infected under-five children receiving ART by identifying significant predictors that have role in mortalities of these age groups by including more proxy variable and segmented to specific age group of under five than under 15 children unlike some studies. 
Methods and materials

Study design and setting: Institution based retrospective follow up study was conducted to children who initiated ART on the period from 2014 to 2019 in Amhara regional state, Oromia 'liyu' zone, Northeast Ethiopia from March 1, 2020 to April 30 2020. According to zonal administrative report, the current estimated number of population is around 585, 968, majority of the society are farmers, the zone contains seven "weredas" and 127 "kebeles" (107 rural and 20 urban). There are two hospitals and twenty-eight health centers, among them more than $90 \%$ of them give PMTCT services and the two hospitals and eight of health centers are giving ART services. Currently there around 3,061 ART taking patients $(\underline{12})$

Study population: All under-five children with HIV infection and taking ART in government health facilities providing ART service on the zone were used as source population. And under five children who started treatment on the period from 2014 to 2019 were study population. Children with incomplete information on predictors; when one of main independent variables (age of the child, age at the start of ART and regimen) are not present in the document were excluded from the study.

Sample size determination and Sampling technique: Sample size was determined by using statistical software called STATA version 14, considering proportional allocation of events using log rank test (freed man principles). Significant predictors of mortality among HIV infected under-five children taking ART were used from different literatures and software calculation computed with considering $95 \% \mathrm{CI}, 80 \%$ power, $5 \%$ level of significance and $10 \%$ assumption of withdrawals giving total sample size of 376 for this study.

All ten health facilities providing ART service were included and a total under five children on treatment on the period from January 2014 to June 2019 were extracted from the registry. At this stage sampling frame was developed and total sample size was compared with the total under

Data collection methods and tool : Data was collected from February 1,2020 to March

12730,2020 by reviewing the patients' medical charts (follow up and ART intake forms) using data 
reviewed published studies. Data on death of HIV positive children while on ART were obtained from providers report on the medical cards. Death at home, after discharge, had been ascertained by the drug adherence counselor (case managers) using contact addresses. The most recent laboratory results before ART initiation were used as baseline values. If there was no pretreatment laboratory test, results obtained within three month of ART initiation was considered as baseline values.

Study variables: Time to death due to HIV/AIDS after initiation of ART is the dependant variable. Socio demographic predictors ( as age of child, sex of child, sex of care giver, age of caregiver, marital status of caregiver, Residence of care giver, place of birth, family status of the child) and Clinical and Immunological predictors (Age at start of ART, WHO clinical stage, TB at baseline, Hemoglobin level, CD4 count, Cotrimoxalzole status, INH status, ART regimen, child adherence, nutritional status, co morbid illness, history of recurrent common childhood infections) are independent variable of the study.

\section{Operational definitions:}

Advanced WHO clinical stages: are stage III and IV baseline clinical stages of HIV-infected under-five children during enrolment to ART (13).

Anemia: Hemoglobin level is below $10 \mathrm{mg} / \mathrm{dl}(\underline{14})$.

Censored: When the child is lost-to-follow up, transferred out, withdrawn from follow-up, death by another causes and lives beyond the study time (14).

CD4 count below threshold: CD4 counts less than 350 cells $/ \mathrm{mm}^{3}$ (15).

Event: death due to HIV infection

Fair adherence to medications: adherence level of under-five children to ART medications within the last three months, between $90-95 \%$ was considered as fair adherence (3).

Mild WHO clinical stages: are stage I and II baseline clinical stages of HIV-infected under-five children during ART enrolment (13).

Moderate malnutrition: Nutritional anthropometry of weight for height is between $70 \%-80 \%$ or height for age Z-score of less than -3 SD or having weight for age Z-score of <-3 SD (16). 
Opportunistic infections: HIV-infected under-five child on ART developed one or more registered history of opportunistic infections during follow-up periods.

Poor adherence to medications: adherence level of under-five children to ART medications within the last three months, between less than $90 \%$ was considered as poor adherence (3).

Recurrent common child hood infection: Having more than three consecutive episodes of diarrhea, pneumonia, upper respiratory tract infections or skin infections.

Severe malnutrition: when nutritional anthropometry of weight for height is less than $70 \%$ or height for age Z-score of less than -3 SD or having weight for age Z-score of <-3 SD (16).

Survival time: the length of time in months a child was followed from the time the child started ART until death, was lost to follow up, or was still on follow up (14).

Data management and analysis: The data was coded and entered into Epi-data version 3.1 and exported to Statistical software package (STATA version 14). Checking for missing values and presence of influential outliers has been done, outliers were managed by taking highest or second smallest values within the data (17). Descriptive statistics such as mean (standard deviation) for normally distributed data, median (inter quartile range), frequencies and proportions was used to describe the cohort. Kaplan-Meier survival curve together with log rank test was used to assess survival experience of an individual at specific times and to compare survival between different independent variables. Schoenfeld residuals test was used to check interaction of each covariate with time and graphical methods was used to check the Cox Proportional Hazard ( $\mathrm{PH})$ assumption. Uni-variable Cox proportional hazards regression was done for each independent variable and outcome of interest to identify potentially significant variables for consideration in the multivariate Cox proportional hazards regression model. Variables whose significance test below 0.25 in uni-variable analysis had been selected for final model. In addition, context and findings of previous studies were considered in the identification of candidate variables for multivariate analysis. The results of the final model were expressed in terms of adjusted hazard ratio (AHR) with 95\% confidence intervals (CI) with significance of $\mathrm{P}<0.05$. The final model goodness fitness was assessed by using cox-snell residual technique. 
A total of 376 under-five children were participated in the study. Nearly half of them, 189 (50.3\%) were male and $243(64.63 \%)$ of children live in rural areas. The median age of participants at ART initiation was $32(\mathrm{IQR}=22-44)$ months (Table1).

Table 1: Socio-demographic characteristics of HIV infected under-five age children on

ART in Oromia 'liyu' Zone, Amhara region, Ethiopia 2020.

\begin{tabular}{|c|c|c|}
\hline Variables & Category & Frequency (\%) \\
\hline \multirow{2}{*}{$\begin{array}{l}\text { Age at start of ART } \\
\text { (in months) }\end{array}$} & $\leq 24$ months & $95(25.27 \%)$ \\
\hline & 25-59 months & $281(74.73 \%)$ \\
\hline \multirow[t]{2}{*}{ Sex } & Male & $189(50.27 \%)$ \\
\hline & Female & $187(49.73 \%)$ \\
\hline \multirow[t]{2}{*}{ Place of birth of the child } & Health institution & $208(55.32 \%)$ \\
\hline & Home & $168(44.68 \%)$ \\
\hline \multirow[t]{4}{*}{ Family status } & Both alive & $239(63.56 \%)$ \\
\hline & Father died & $61(16.22 \%)$ \\
\hline & Mother died & $30(7.98 \%)$ \\
\hline & Orphaned & $46(12.23 \%)$ \\
\hline \multirow[t]{2}{*}{ Sex of care giver } & Male & $91(24.20 \%)$ \\
\hline & Female & $285(75.80 \%)$ \\
\hline \multirow[t]{3}{*}{ Age of care giver } & $<20$ years & $6(1.60 \%)$ \\
\hline & 20-35 years & $162(43.09 \%)$ \\
\hline & $>35$ years & $208(55.32 \%)$ \\
\hline \multirow[t]{2}{*}{ Place of residence } & Urban & $133(35.37 \%)$ \\
\hline & Rural & $243(64.63 \%)$ \\
\hline \multirow[t]{4}{*}{ Marital status of care giver } & Married & $256(68.09 \%)$ \\
\hline & Single & $36(9.57 \%)$ \\
\hline & Divorced & $37(9.84 \%)$ \\
\hline & Widowed & $47(12.58 \%)$ \\
\hline
\end{tabular}

Based on the study a huge segment of underfive were delivered at home168 (44.68\%). 


\section{Baseline clinical characteristics}

194 One hundred fifty $(39.89 \%)$ of HIV infected under five children born from mothers who do not

195 initiated PMTCT service during their period of pregnancy. Based on WHO clinical staging, 92 196 (24.47\%) of them initiated ART at advanced stage (clinical stage III and stage IV).

197 Table 2: Baseline clinical characteristics of under-five children on ART in Oromia 'liyu' 198 Zone, Amhara region, Ethiopia 2020.

\begin{tabular}{|c|c|c|}
\hline Variable & Category & Frequency $(\%)$ \\
\hline \multirow[t]{2}{*}{ Maternal history of PMTCT } & Yes & $226(60.11 \%)$ \\
\hline & No & $150(39.89 \%)$ \\
\hline \multirow[t]{3}{*}{ Baseline Nutritional status } & Severe malnutrition & $38(10.11 \%)$ \\
\hline & Moderate malnutrition & $138(36.97 \%)$ \\
\hline & $\begin{array}{l}\text { Normal nutritional } \\
\text { status }\end{array}$ & $199(52.93 \%)$ \\
\hline \multirow{2}{*}{$\begin{array}{l}\text { History of OI's during follow } \\
\text { up }\end{array}$} & Yes & $150(39.89 \%)$ \\
\hline & No & $226(60.11 \%)$ \\
\hline \multirow[t]{3}{*}{ Adherence status (child) } & Good & $280(74.47 \%)$ \\
\hline & Fair & $68(18.09 \%)$ \\
\hline & Poor & $28(7.45 \%)$ \\
\hline \multirow[t]{2}{*}{ Baseline WHO staging } & Mild stages & $284(75.53 \%)$ \\
\hline & Advanced stages & $92(24.47 \%)$ \\
\hline \multirow[t]{2}{*}{ Baseline CD4 count } & Below threshold & $68(22.74 \%)$ \\
\hline & Above threshold & $231(77.26 \%)$ \\
\hline \multirow[t]{2}{*}{ Baseline hemoglobin } & $<11 \mathrm{mg} / \mathrm{dl}$ & $109(28.99 \%)$ \\
\hline & $\geq 11 \mathrm{mg} / \mathrm{dl}$ & $267(71.01 \%)$ \\
\hline \multirow[t]{2}{*}{ Cotrimoxalzole prophylaxis } & Yes & $258(68.62 \%)$ \\
\hline & No & $118(31.38 \%)$ \\
\hline \multirow[t]{2}{*}{ INH prophylaxis } & Yes & $226(60.11 \%)$ \\
\hline & No & $150(39.89 \%)$ \\
\hline
\end{tabular}

Nearly half of children accounting 199 (52.93\%) had normal nutritional status, where as 138

$200(36.97 \%)$ and $38(10.11 \%)$ of them had either moderate or severe malnutrition respectively. 
201

202

203

204

205

206

207

208

209

210

211

212

213

214

215

216

217

218

219

220

221

222

223

224

225

226

227

228

During the study follow up period $280(74.47 \%)$ and $68(18.09 \%)$ of participants have good and fair adherence, respectively; in other side 28 (7.45\%) of them were with poor adherence, of them $9(32 \%)$ of them had died at the end of the study. The baseline CD4 count were available for more than three fourth $(299(79.5 \%))$ of under-five children, around $68(22.74 \%)$ of them had CD4 level less than threshold ( $<350$ cells $\left./ \mathrm{mm}^{3}\right)$ and remaining $231(77.26 \%)$ of them had CD4 level above threshold (>350 cells $/ \mathrm{mm}^{3}$ ) $($ Table 2$)$.

\section{Survival characteristics after initiation of ART:}

After initiation of ART, HIV-infected children were followed for 3 months to 41 months and median follow up period was 19 months $(\mathrm{IQR}=11-26.8)$. At the end of follow up period, 285 $(80.28 \%)$ of the children were alive, 39 (10.99\%) were lost to follow up, 11 (3.94\%) were transferred out to other health facilities, $4(1.13 \%)$ were dead by other case and $21(5.59 \%)$ were reported dead due to HIV/AIDS. Of the 21 deaths, two (9.5\%) of them occurred within the first three months after initiation of HAART and 9 (42.85\%) of them occurred within the first 12 months of ART initiation. The incidence of death was 5.9 deaths per 100 child-months (95\%CI: 3.89-9.09) during the follow-up period. The overall mean time to death was 19.7 months $(95 \% \mathrm{CI}=18.74-20.67)$. The median estimated survival time of under-five age children after initiation of ART in the study was 19 months (IQR=11.00-26.80). The cumulative probability of survival of under-five children on ART after last month of follow-up was $87.23 \%$ (95\%CI; 79.42-92.22). The cumulative survival probabilities of children after 3, 6, 12, 24 and 36 months were $0.99,0.98,0.97,0.89$ and 0.87 respectively.

\section{Comparison of survival curves}

The overall Kaplan-Meier survivor function estimate shows that most deaths had been occurred in the earlier months of ART initiation. Based on the findings, from the total of 21 deaths, $2(9.5 \%)$ of them occurred in the first six months of follow-up and around $9(42.9 \%)$ deaths occurred in the first 12 months of follow-up, which became declining through follow up time and continues steadily at later months of follow up.

Some graphs compared to other covariates have shown relatively larger gaps between categories, such as drug adherence, baseline nutritional status and WHO clinical stages of the children. 


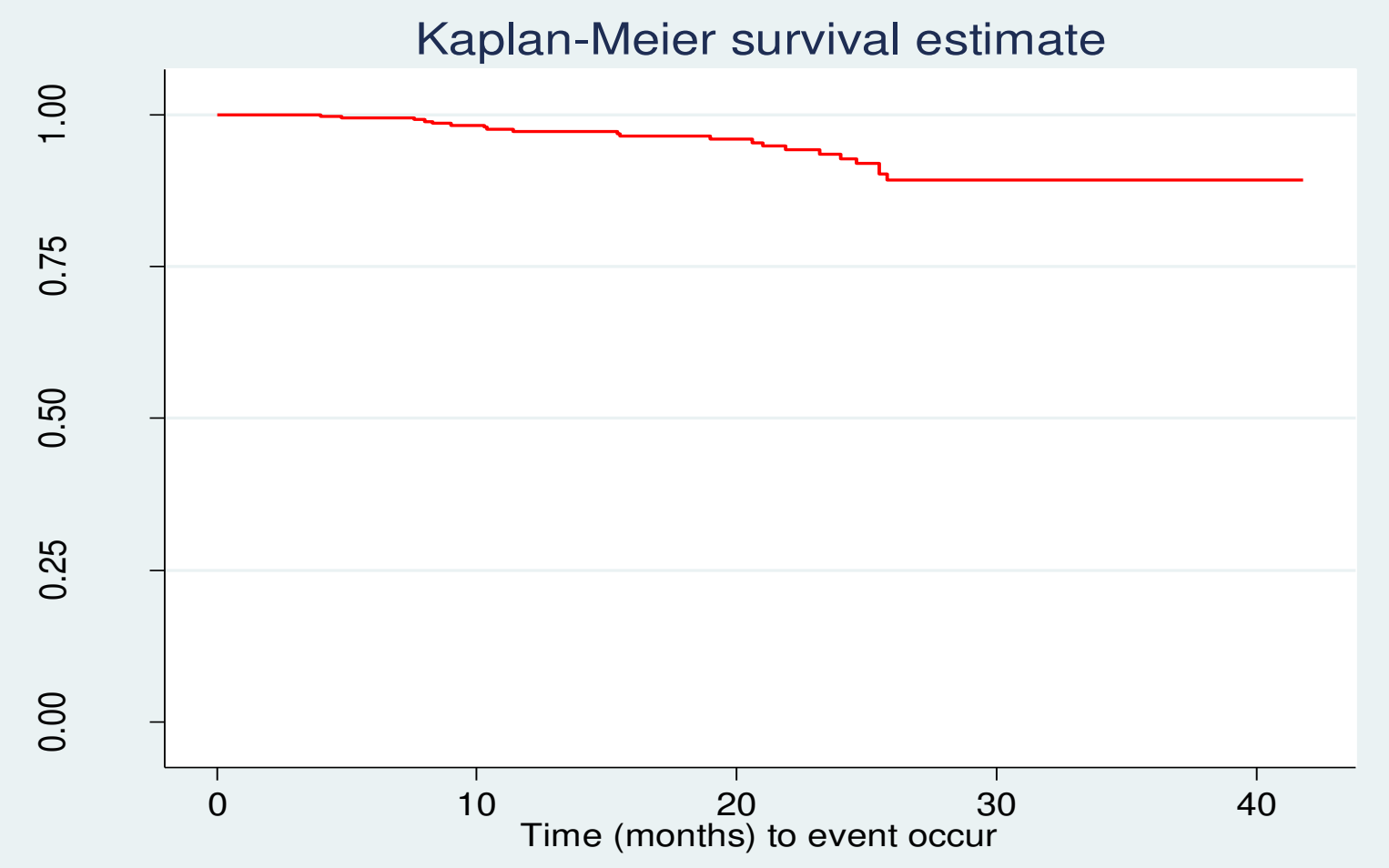

229

Figure 1: Plot of overall of Kaplan-Meier survivor function among under-five children on ART in Oromia 'liyu' Zone, Amhara region, Ethiopia 2020.

Based on the results from Kaplan-Meier survival curve, significant covariates (Baseline WHO stage, Drug adherence and nutritional status of the child) have shown certain pattern in outcome event (death) of under-five children throughout the follow up time (Figure 1).

Under-five children with severe malnutrition during initiation of ART have developed death in early months of follow up significantly compared to normal and moderate malnutrition. Children with WHO advanced clinical stage (III and IV) have survived less than children with mild stages (I and II), this observed difference was also statistically significant ( $\mathrm{p}<0.001)$. Children with good adherence have longer survival experience than those children with fair and poor adherence and those under-five children with poor adherence have lower survival experience compared to fair once, these differences have been statistically significant ( $\mathrm{p}<0.001)$ (Figure 2-Figure 5).

The log-rank value of $p<0.001$ suggests that there was a difference in probability of developing death between groups throughout the follow-up period. The Kaplan-Meier survival curve remarkably decreases for children with severe malnutrition. 


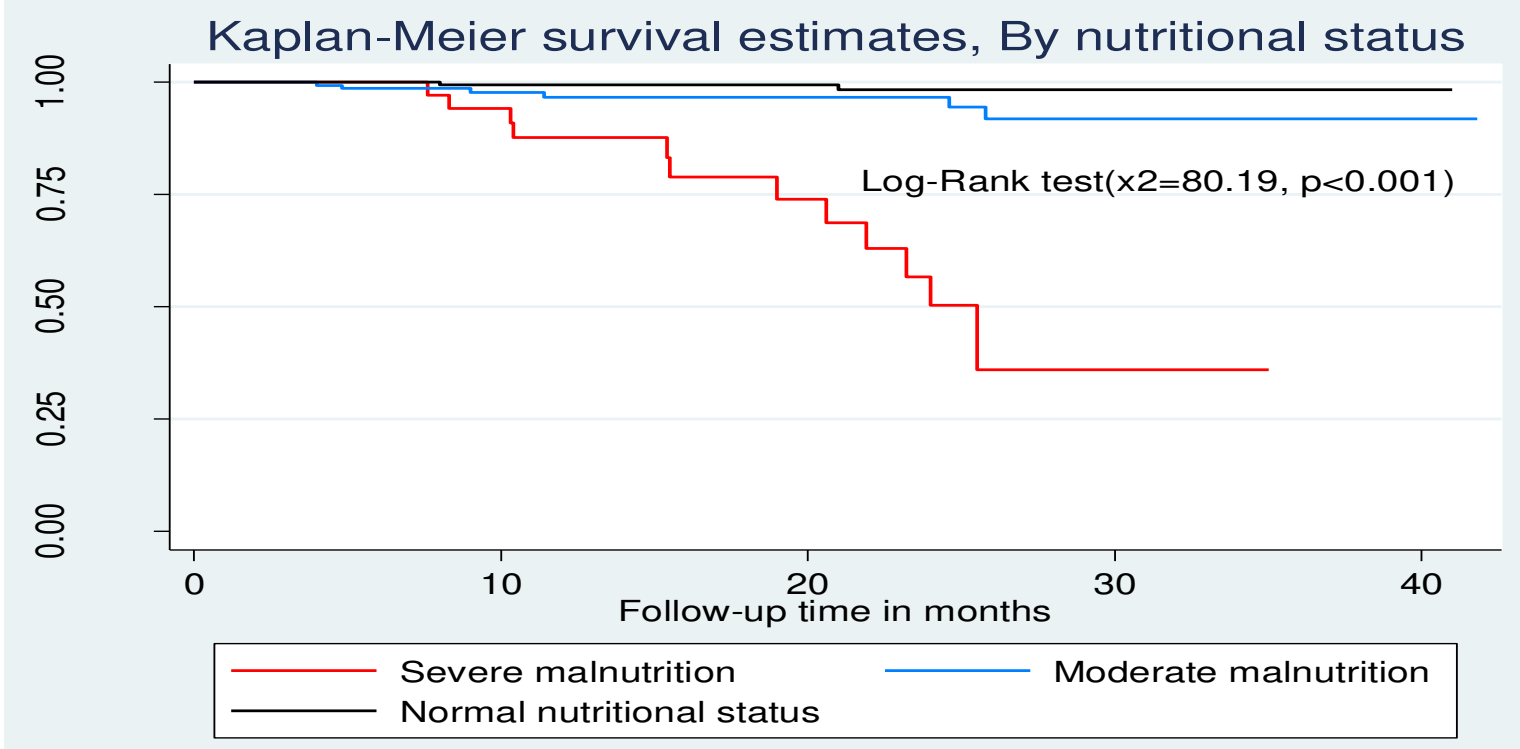

246 Figure 2: Kaplan-Meier survival estimates of baseline nutritional status among under-five children on 247 ART in Oromia 'liyu’ Zone, Amhara region, Ethiopia 2020.

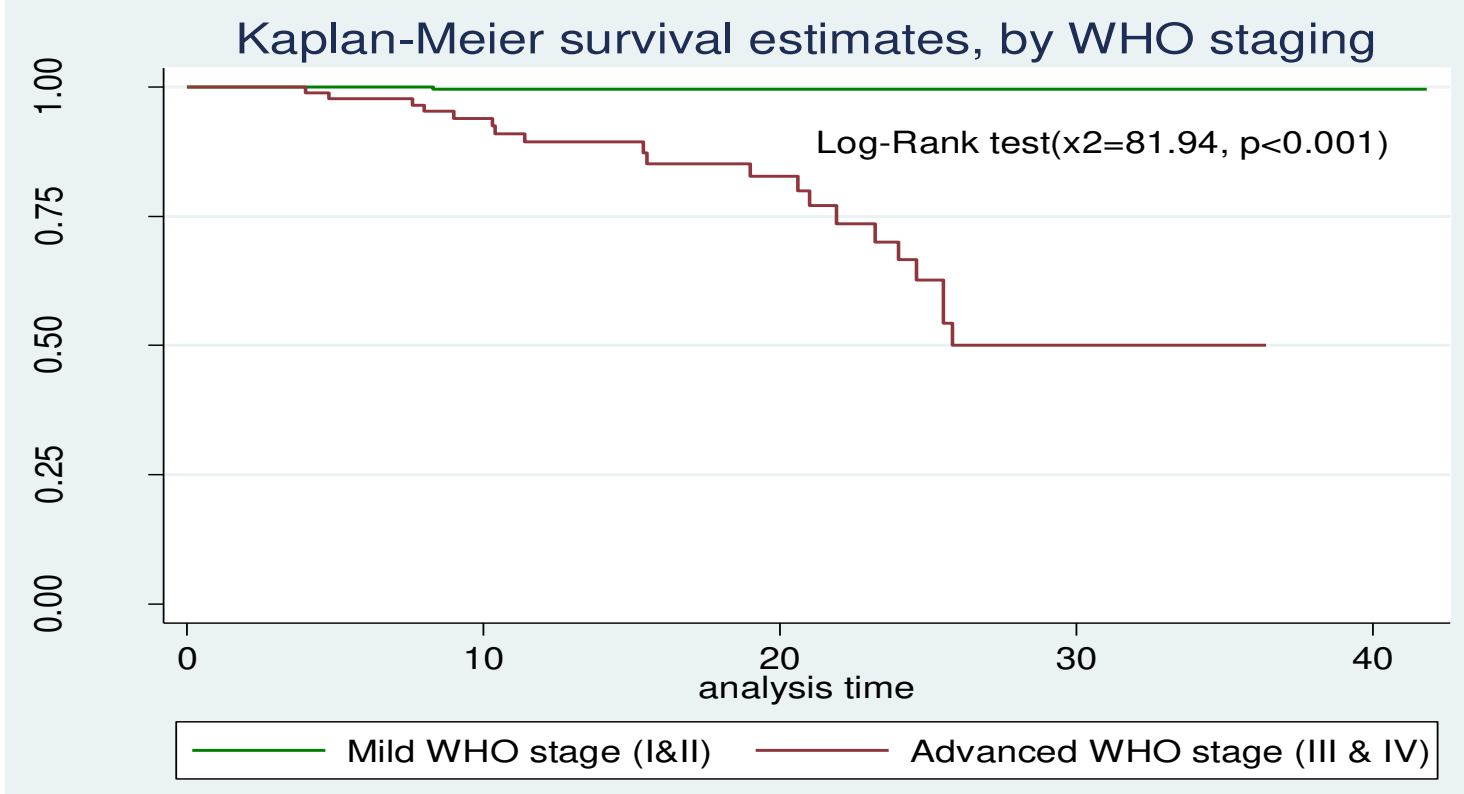

259 Figure 3: Kaplan-Meier survival estimates of baseline WHO staging among under-five children on ART 260 in Oromia 'liyu' Zone, Amhara region, Ethiopia 2020. 


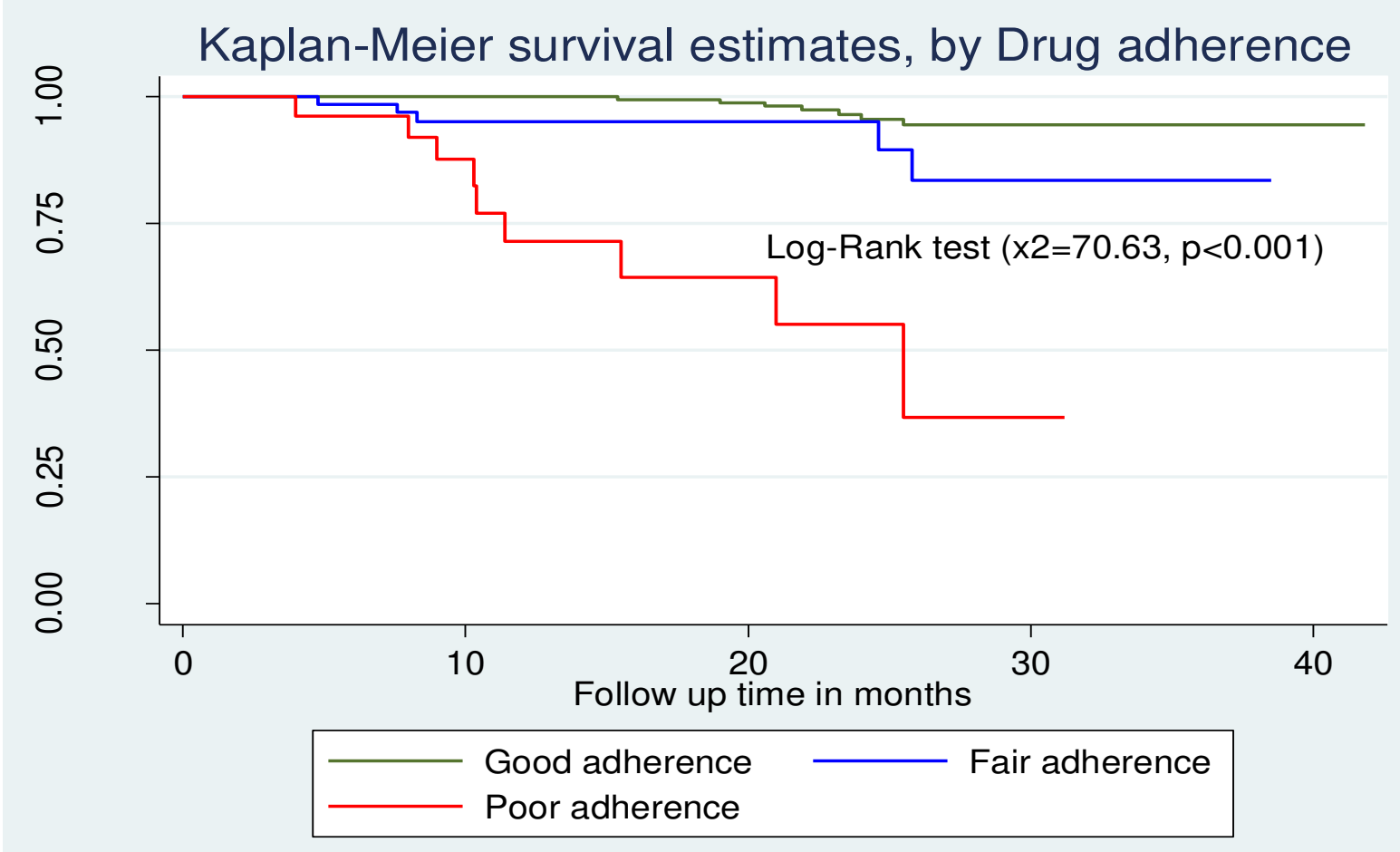

262 Figure 4: Kaplan-Meier survival estimates based on adherence status among under-five children on ART in Oromia 'liyu' Zone, Amhara region, Ethiopia 2020.

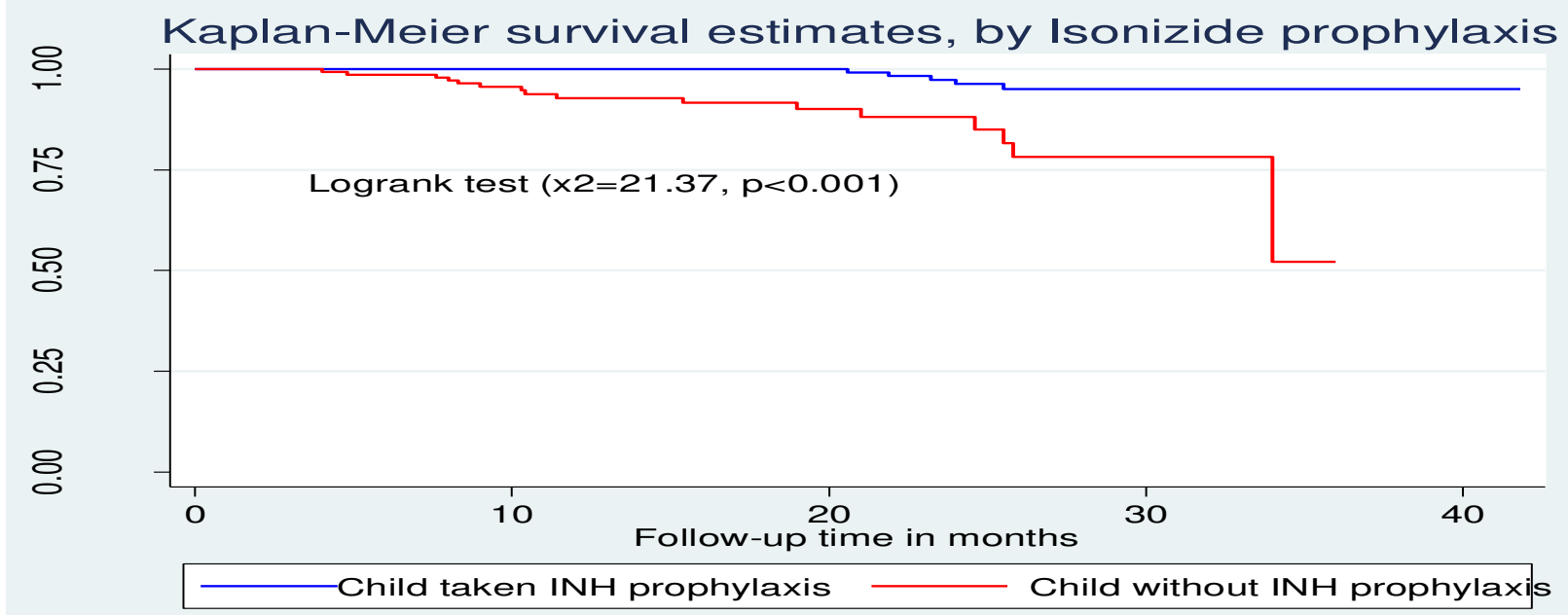

Figure 5: Kaplan-Meier survival estimates based on their Isoniazide prophylaxis among under266 five children on ART in Oromia 'liyu' Zone, Amhara region, Ethiopia 2020. 


\section{Predictors of HIV-infected under-five age child death}

268 On uni-variable Cox-regression analysis: age of the child, birth place of the child, family status 269 of the child, age of caregiver, marital status of caregiver, maternal history of PMTCT during 270 pregnancy, baseline WHO clinical stage of the child, nutritional status of the child at enrolment, 271 history of opportunistic infection during follow up, child adherence to medications, child status 272 of cotrimoxalzole and Isoniazide prophylaxis during their infancy period were associated with 273 outcome variable at p-value $<0.25$.

274 P value $<0.25$ was considered for multivariable Cox-regression to recruit potential determinant 275 covariates of HIV-infected under-five child death. Baseline nutritional status of the child, Child 276 adherence to ART medications during follow up, Baseline clinical stage of the child during ART 277 initiation and status of INH-prophylaxis after birth were identified as independent determinants 278 of death in under-five children.

279 The nutritional status of HIV-infected under-five children was found to be predictors of death, in 280 which the risk of death at any given time during the study period among under-five children with 281 severe malnutrition was almost five (AHR=4.93; 95 CI: 1.04, 10.47) times higher than those 282 children who had normal nutritional status. 
Table 3: Multivariable Cox-regression analysis of predictors of death among under-five children on ART at Amahara regional state, Oromia "liyu" zone, Northeast Ethiopia 2020

\begin{tabular}{|c|c|c|c|c|c|c|c|}
\hline \multirow[t]{2}{*}{ Covariates } & \multirow[t]{2}{*}{ Categories } & \multicolumn{2}{|c|}{ Outcome } & \multirow[t]{2}{*}{ CHR } & \multirow[t]{2}{*}{ AHR } & \multirow[t]{2}{*}{ CI (95\%) } & \multirow{2}{*}{$\begin{array}{l}P \text { - } \\
\text { values }\end{array}$} \\
\hline & & Dead & Censored & & & & \\
\hline \multirow{6}{*}{$\begin{array}{c}\text { Baseline } \\
\text { nutritional } \\
\text { status }\end{array}$} & Normal & 2 & 197 & 1 & 1 & & \\
\hline & $\begin{array}{l}\text { nutritional } \\
\text { status }\end{array}$ & $(1.11 \%)$ & $(98.99 \%)$ & & & & \\
\hline & Moderate & 6 & 133 & 4.79 & 2.07 & {$[0.41,10.39]$} & 0.376 \\
\hline & malnutrition & $(4.32 \%)$ & $(95.68 \%)$ & & & & \\
\hline & Severe & 13 & 25 & 9.78 & 4.93 & {$[1.04,10.47]$} & 0.043 \\
\hline & malnutrition & $(34.21 \%)$ & $(65.79 \%)$ & & & & \\
\hline \multirow{6}{*}{$\begin{array}{l}\text { Adherence } \\
\text { status of } \\
\text { the child }\end{array}$} & Good & 7 & 273 & 1 & 1 & & \\
\hline & & $(2.50 \%)$ & $(97.50 \%)$ & & & & \\
\hline & Fair & 5 & 63 & 3.56 & 2.25 & {$[0.70,7.30]$} & 0.175 \\
\hline & & $(7.35 \%)$ & $(92.65 \%)$ & & & & \\
\hline & Poor & $9(32.14 \%)$ & 19 & 12.84 & 3.86 & {$[1.37,10.88]$} & 0.011 \\
\hline & & & $(67.86 \%)$ & & & & \\
\hline \multirow{4}{*}{$\begin{array}{l}\text { Baseline } \\
\text { WHO } \\
\text { Clinical } \\
\text { stages }\end{array}$} & Mild & 1 & 283 & 1 & 1 & & \\
\hline & (stage I \& II) & $(0.35 \%)$ & $(99.65 \%)$ & & & & \\
\hline & Advanced & 20 & 72 & 25 & 6.37 & {$[3.33,10.14]$} & 0.002 \\
\hline & (stage III\&IV) & $(21.74 \%)$ & $(78.26 \%)$ & & & & \\
\hline \multirow{4}{*}{$\begin{array}{l}\text { INH } \\
\text { prophylaxis }\end{array}$} & Yes & 5 & 221 & 1 & 1 & & \\
\hline & & $(2.21 \%)$ & $(97.79 \%)$ & & & & \\
\hline & No & 16 & 134 & 7.99 & 3.55 & {$[1.24,10.18]$} & 0.018 \\
\hline & & $(10.67 \%)$ & $(89.33 \%)$ & & & & \\
\hline
\end{tabular}

The risk of death among under-five children with poor adherence to ART medications is almost

287 four $(\mathrm{AHR}=3.9$; CI: 1.37, 10.88) times higher than those children with good adherence. In 288 addition, the risk of death among under-five children with advanced WHO clinical stages (III 289 and IV) at enrolment to ART was almost seven times (AHR=6.56; CI: 3.33, 12.14) higher hazard 290 of death than children with mild stages (I and II). In other hand, the risk of death in under-five 291 children without history of INH prophylaxis during their age of infancy was four times $292 \quad(\mathrm{AHR}=3.6 ; \mathrm{CI}: 1.24,10.18)$ higher than those children with INH prophylaxis. (Table 3) 
294 In this study, high number of deaths 9(42.9\%) occurred within the first one year, after starting ART. This finding is similar to the studies conducted in Ethiopia, Harar (15), Gonder, Addis $\operatorname{Ababa(18)}$ Debretabor and Desse(13)), Ngeria(19), Cameroon(20) and study in Sub-Saharan Africa(21), reporting that the death rate was high in early times of ART initiation. Higher death in this period of time could be resulted from almost same health service coverage and late utilization of ART services in the countries.

One of significant associated predictors of death among HIV-infected under-five children on ART was baseline nutritional status of the child during enrolment, by which children with severe malnutrition status at beginning ART showed to have shorter duration of survival than the normal once $(\mathrm{AHR}=4.9 ; 95 \mathrm{CI}: 1.04,23.52)$. This finding is consistent with studies done in Ethiopia (Amhara region, Debretabor and Desse) $(\underline{13}, \underline{16})$. This similarity might be due to the fact that children with poor nutritional status may clinically manifest poor outcomes to treatments, since their immunity is compromised by lack sufficient nutrients to their cells; In other hand study in Cameroon explained poor nutritional status is not a cause for increased rate of HIV-infected children(20) this might be because of difference in socio-economic and nutritional culture between the two countries.

WHO clinical stage during enrolment to ART was other covariates that had significant effect on mortality of under-five child. The hazard of death on those children with advanced stages (stage III and IV) at ART enrolment was around six times higher than those with mild stage (stage I and II). This finding was consistent with studies done in Ethiopia, Amahra region(16), Gonder(18) and Addis Ababa(22) and the finding is also consistent with studies done in Cameroon (20) and southern Africa(23). This similarity might be because of children with higher stages have weaker immune status and are highly vulnerable to OIs that may lead to early occurrence of death. In this study from total 92 children with advanced stages at ART enrolment 65 (70.7\%) of them had developed one or more OIs during follow up period and from total of 21 deaths during the study period 19 (90.5\%) had developed death. In contrast, a study done in Southern regional state (Gamo-Gofa) (14) and Amhara regional state (Desse and Debretabor Hspitaals) (13), on children survival after initiation of ART, found as if WHO clinical stages as non-significant predictor of HIV-infected child death. The reason for this difference might be level of community awareness 
323 towards early utilization of ART services between the areas, in order to avert child death before

324 development of series complication.

325 Adherence status of the child to medications throughout the follow-up time is impotant predictor

326 in agreement with majority of studies. INH prophylaxis status under-five children during their

327 age of infancy were among one of significant determinants of death. Children with no history of

328 INH prophylaxis were associated with reduced survival. This finding was consistent with

329 findings in a study done around Gamo-Gofa (Ethiopia) (14). The cause for this might be due to 330 reduced level of early identification of HIV-infected children and low coverage of PMTCT in

331 between this two places and the whole country (Ethiopia). The possible reason for such higher

332 level of death on these under-five children without INH prophylaxis during ART enrolment

333 could be due occurrence of some OIs like pneumocystis pneumonia, toxoplasmosis, tuberculosis

334 and recurrent diarrheal diseases. In contrast a study done in Addis Ababa on treatment outcomes

335 of HIV-infected children (24) showed that taking INH prophylaxis during has no significant

336 effect on the mortality of ART under-five children, this might be due to difference in

337 accessibility, availability and utilization of health institutions in Addis Ababa and Gamo-Gofa.

338 Conclusion

339 The incidence of death is 5.9 deaths per 100 child-months and the overall mean time to death 340 among under five children on ART is 19.7 months $(95 \% \mathrm{CI}=18.74-20.67)$. The cumulative 341 probability of survival of under-five children on ART after last month of follow-up was $87.23 \%$ 342 i.e. nearly the indicated percentage of children on ART can pass and celebrate the five year of 343 life. The major factors that determine time to death of under-five children with HIV/AIDS on 344 ART were; Baseline nutritional status, WHO clinical stage of the child during ART enrolment, 345 adherence status of the child to medications and INH prophylaxis during infancy period of the 346 child.

347 Acronyms and Abbreviations

3TC Lamuvidine

AIC Akaike-Information Criteria

AHR Adjusted Hazard Ratio

AIDS Acquired Immune Deficiency Syndrome 
ART

AZT

$\mathrm{CDC}$

CHR

$\mathrm{EFV}$

GC

HAART

HIV

INH

MTCT

NVP

OI

PPSS

$\mathrm{PH}$

PMTCT

SDG

TB

TDF

UNAIDS

VIF

WHO
Anti Retroviral Therapy

Zidovudine

Communicable Diseases Control

Crude Hazard Ratio

Efavirenz

Gregorian-Calendar

Highly Active Anti Retroviral Therapy

Human Immune Deficiency

Iso Nicotinyl Hydrazide

Maternal to Child Transmission

Neverapine

Opportunistic Infections

Pediatric Psycho Social Support

Proportional Hazard

Prevention of Mother To Child Transmission

Sustainable Development Goal

Tuberculosis

Tenofovir

United Nations Program on Acquired Immune Deficiency Syndromes

Variance Inflation Factor

World Health Organization 
349 Ethical approval and consent participate: Ethical approval was secured from the Debremarkos 350 University college of Health sciences ethical review office and zonal health offices, and no 351 individual consent is required as the study depend on secondary data with anonymity, and had 352 approval from the zonal health office. All aspects of the study methods and other steps were 353 conducted in accordance with Debremarkos University research regulation guideline and 354 approved ethical clearance. Bati zone health office ethical review committee approved that 355 consent is not applicable and waived for this study.

356 Consent for Publication: Not applicable

357 Data availability and materials: All necessary information is incorporated in the study.

358 Competing interest: The authors declare that we have no conflict of interest with anyone related 359 to this study.

360 Funding: The study was funded by Debremarkos University. However the organization has no 361 role in preparation of manuscript and publication process.

362 Author's contribution: SA and TM contributed from the inception of study, follow of data 363 collection and analysis of data and preparation of the manuscript. YB had role in data collection, 364 analysis and write-up of the manuscript.

365 Acknowledgment: We greatly acknowledge] Zonal and Wereda health office workers and ART 366 focal persons in the health centers and hospitals for their active cooperation during data 367 collection. We are also thankful for Tewodros Eshetie (Ass.Pro of Biostatics and health 368 informatics) for sharing us his pearls of wisdom and comments on this study. 
371 1. Chenneville T. A Clinical Guide to Pediatric HIV: Bridging the Gaps Between Research and

372 Practice: Springer; 2017.

$3732 . \quad$ Avert. Global information and education on HIV and AIDS (Children, HIV and AIDS) Avert.org;

3742019 [cited 2019].

$3753 . \quad$ Federal democratic republic of Ethiopia moh. National guidlines for HIV prevention, care and

376 treatment. Addis Ababa, Ethiopia: Ministry of health; 2018. p. 1-75.

377 4. HIV/AIDS JUNPo. Children and HIV fact sheet. Geneva: UNAIDS. 2016.

378 5. CDC P. Helping babies born to HIV-positive mothers in sub-saharan africa stay healthy and free 379 from HIV. USA2018.

380 6. Hug L, Sharrow D, You D. Levels \& trends in child mortality: report 2017. Estimates developed by

381 the UN Inter-agency Group for Child Mortality Estimation. 2017.

382 7. UNAIDS. Global HIV statistics, Fact sheet_world AIDS day 20192019 [cited 2019].

383 8. WHO. Health topics 2019.

384 9. UNICEF Emof. National Situation Analysis of Children and Women in Ethiopia. Addis Ababa, 385 NCC/UNICEF2019.

10. Estimation UNI-aGfCM, Hug L, Sharrow D, Zhong K, You D, Unicef, et al. Levels \& Trends in Child Mortality: Report 2018, Estimates Developed by the: United Nations Children's Fund; 2018.

11. Federal HIV/AIDS prevention and control office E. HIV prevention in Ethiopia, Natioanl road map 2018-2020. In: Federal HIV/AIDS prevention and control office E, editor. Addis Ababa, Ethiopia: Federal ministry of health, Eethiopia; 2018. p. 1-15.

12. Amhara region Olzho, Ethiopia. Oromia 'liyu' zone health office, annnual report, 2018 Kemise, Ethiopia2018.

13. Arage G, Assefa M, Worku T, Semahegn A. Survival rate of HIV-infected children after initiation of the antiretroviral therapy and its predictors in Ethiopia: A facility-based retrospective cohort. SAGE open medicine. 2019;7:2050312119838957.

14. Sidamo NB, Hebo SH. Survival time and its predictors among HIV-infected children after antiretroviral therapy in public health facilities of Arba Minch town, Gamo Gofa Zone, Southern Ethiopia. Ethiopian Journal of Health Development. 2018;32(2).

15. Edessa D, Asefa F, Sheikahmed J. Early mortality among HIV-positive children initiated antiretroviral therapy in eastern Ethiopia: a retrospective cohort study. Science, Technology and Arts Research Journal. 2015;4(2):157-63.

16. Alebel A, Wagnew F, Tesema C, Kibret GD, Petrucka P, Eshite S. Effects of undernutrition on survival of human immunodeficiency virus positive children on antiretroviral therapy. Italian journal of pediatrics. 2018;44(1):29.

405 17. Kwak SK, Kim JH. Statistical data preparation: management of missing values and outliers. Korean journal of anesthesiology. 2017;70(4):407.

18. Andargie AA, Asmleash Y. Survival time of human immunodeficiency virus (HIV) infected children under 15 years of age after initiation of antiretroviral therapy in the University of Gondar Comprehensive Specialized Hospital, Ethiopia. Journal of AIDS and HIV Research. 2018;10(4):49-55.

19. Anigilaje EA, Aderibigbe SA. Mortality in a cohort of HIV-infected children: a 12-month outcome of antiretroviral therapy in Makurdi, Nigeria. Advances in medicine. 2018;2018.

20. Nlend AN, Loussikila A. Predictors of mortality among HIV-infected children receiving highly active antiretroviral therapy. Medecine et maladies infectieuses. 2017;47(1):32-7.

21. Ahmed I, Lemma S. Mortality among pediatric patients on HIV treatment in sub-Saharan African countries: a systematic review and meta-analysis. BMC public health. 2019;19(1):149.

417 22. Mulugeta A, Assefa H, Tewelde T, Dube L. Determinants of survival among HIV positive children on antiretroviral therapy in public hospitals, Addis Ababa, Ethiopia. Qual Prim Care. 2017;25(4):235-41. 
418 23. Porter M, Davies M-A, Mapani MK, Rabie H, Phiri S, Nuttall J, et al. Outcomes of infants starting 419 antiretroviral therapy in Southern Africa, 2004-2012. Journal of acquired immune deficiency syndromes 420 (1999). 2015;69(5):593.

421 24. Mokgatle MM, Abasho DC. Treatment outcomes of antiretroviral therapy among pediatric

422 patients in Zewditu memorial hospital, Addis Ababa, Ethiopia. PULA: Botswana J Afr Stud. 2016;30(1):11. 
Figures

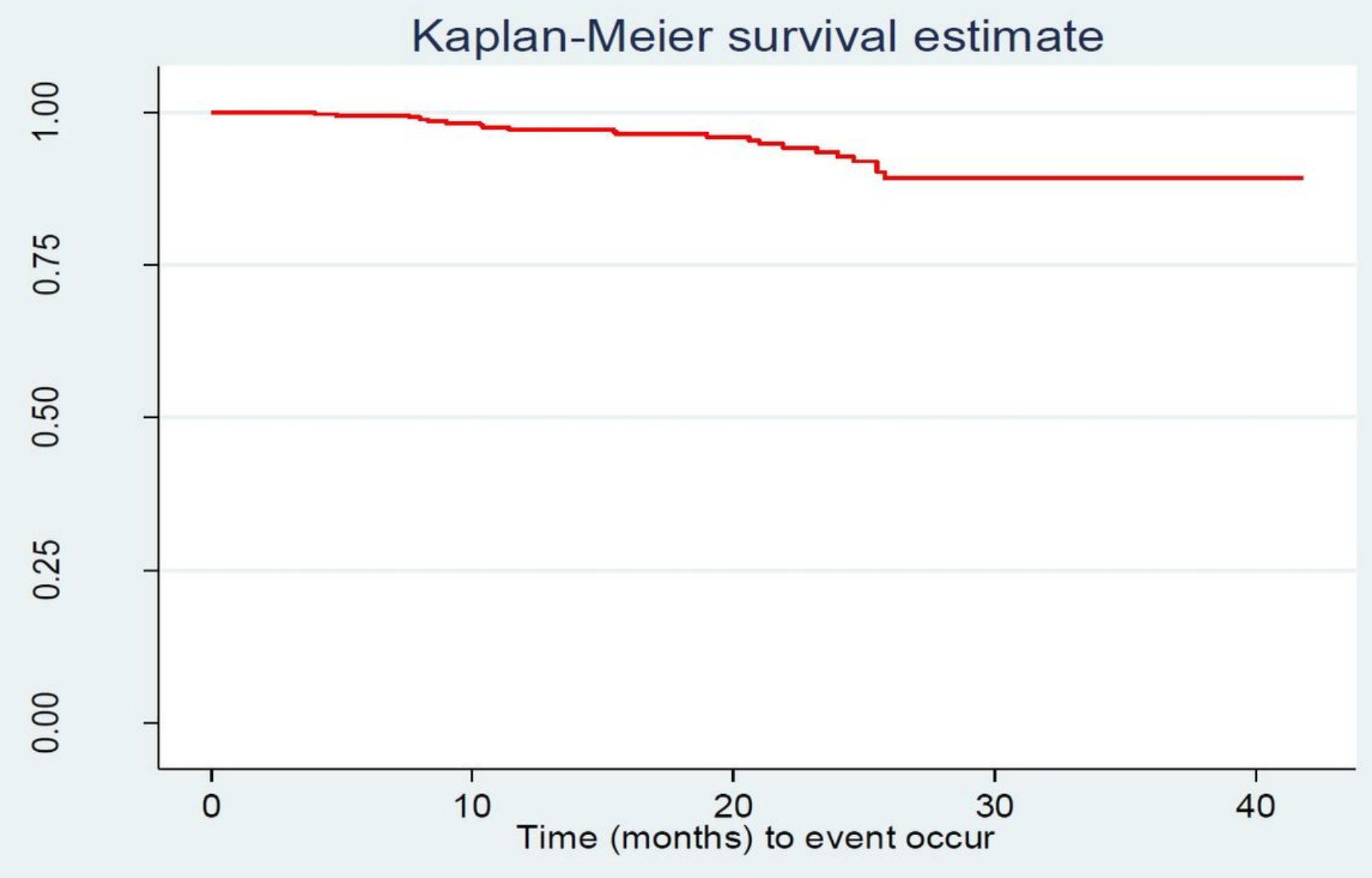

Figure 1

Plot of overall of Kaplan-Meier survivor function among under-five children on ART in Oromia 'liyu' Zone, Amhara region, Ethiopia 2020. 


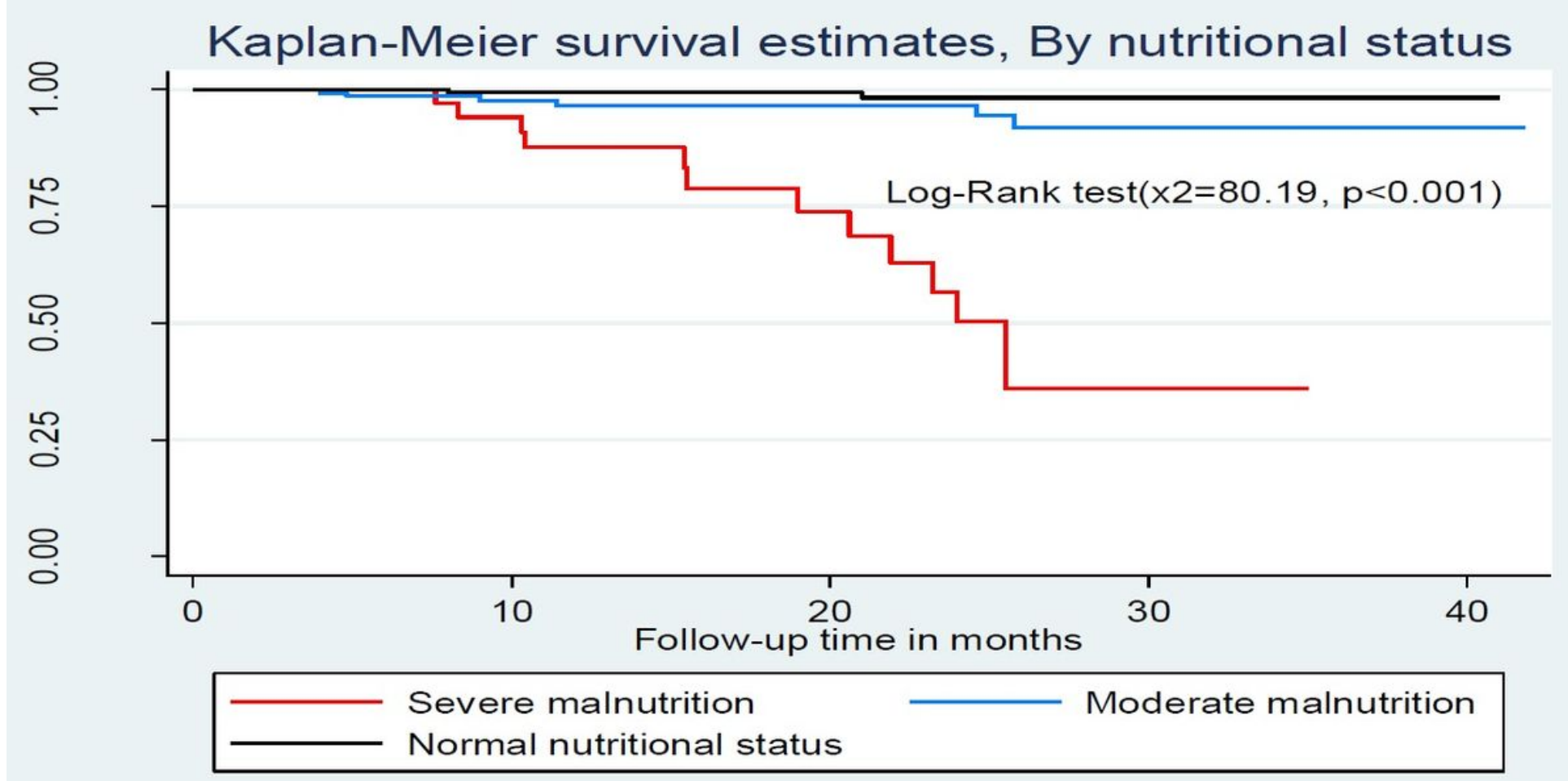

Figure 2

Kaplan-Meier survival estimates of baseline nutritional status among under-five children on ART in Oromia 'liyu' Zone, Amhara region, Ethiopia 2020.

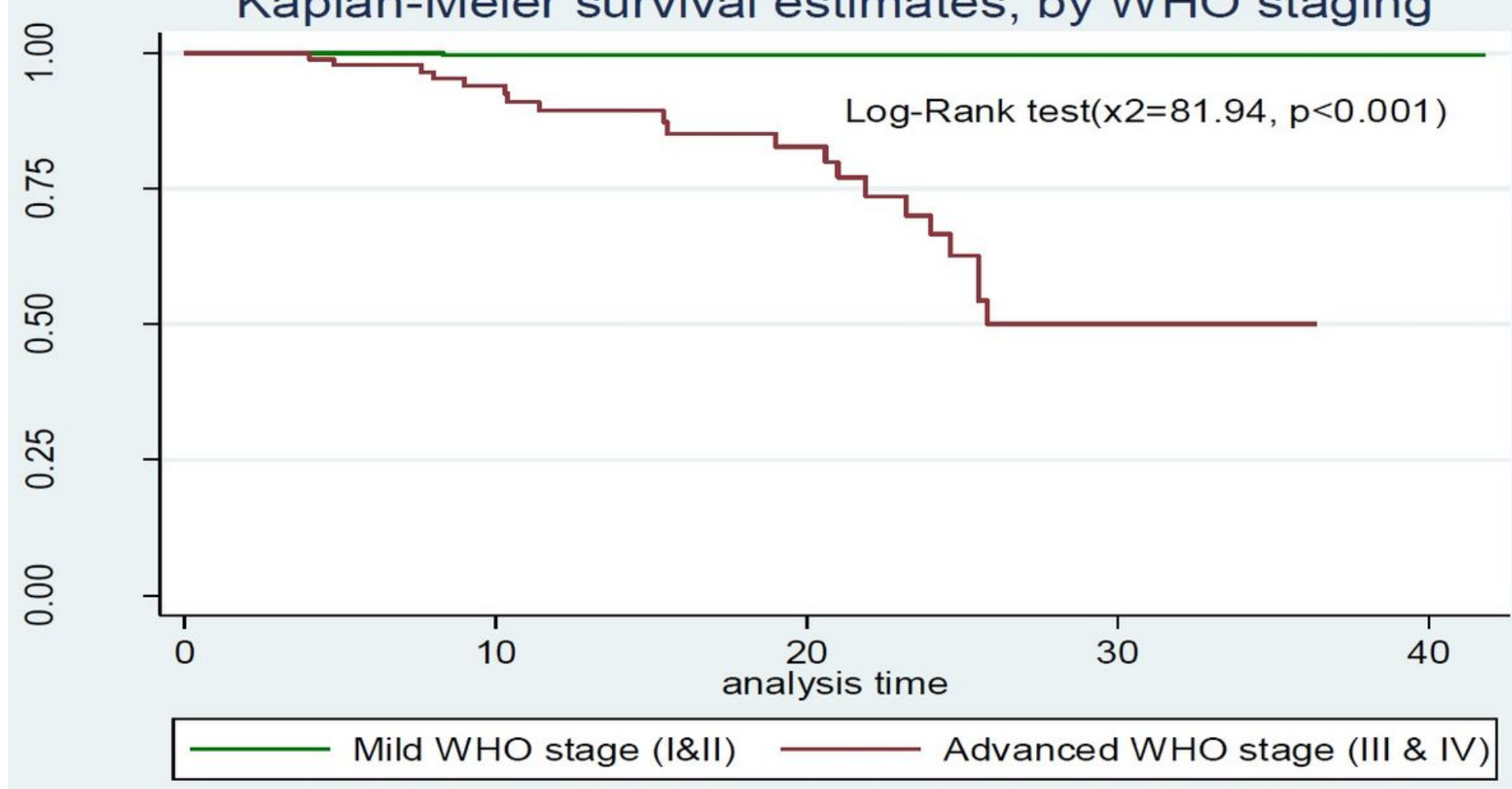


Figure 3

Kaplan-Meier survival estimates of baseline WHO staging among under-five children on ART in Oromia 'liyu' Zone, Amhara region, Ethiopia 2020.

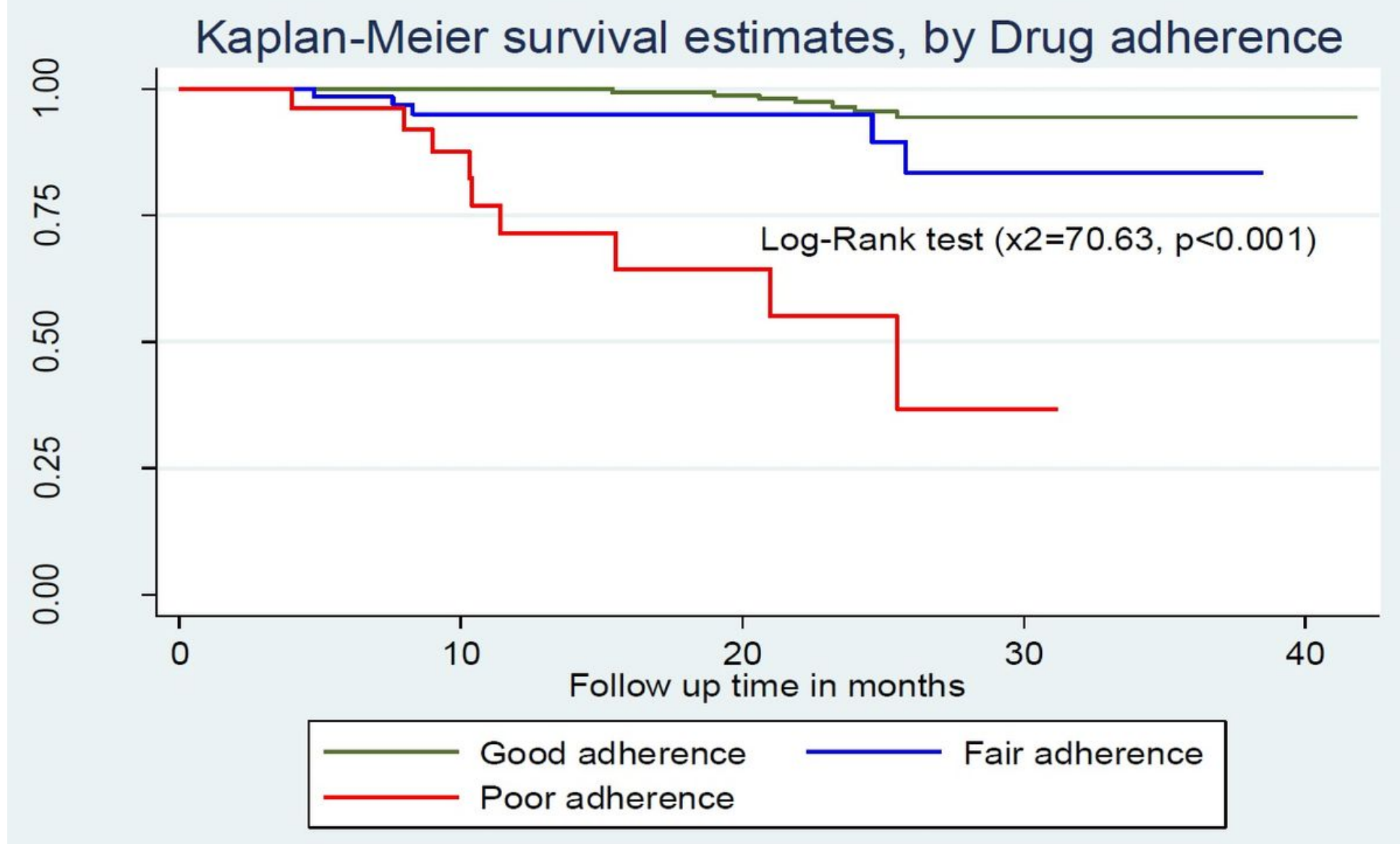

Figure 4

Kaplan-Meier survival estimates based on adherence status among under-five children on ART in Oromia 'liyu' Zone, Amhara region, Ethiopia 2020. 


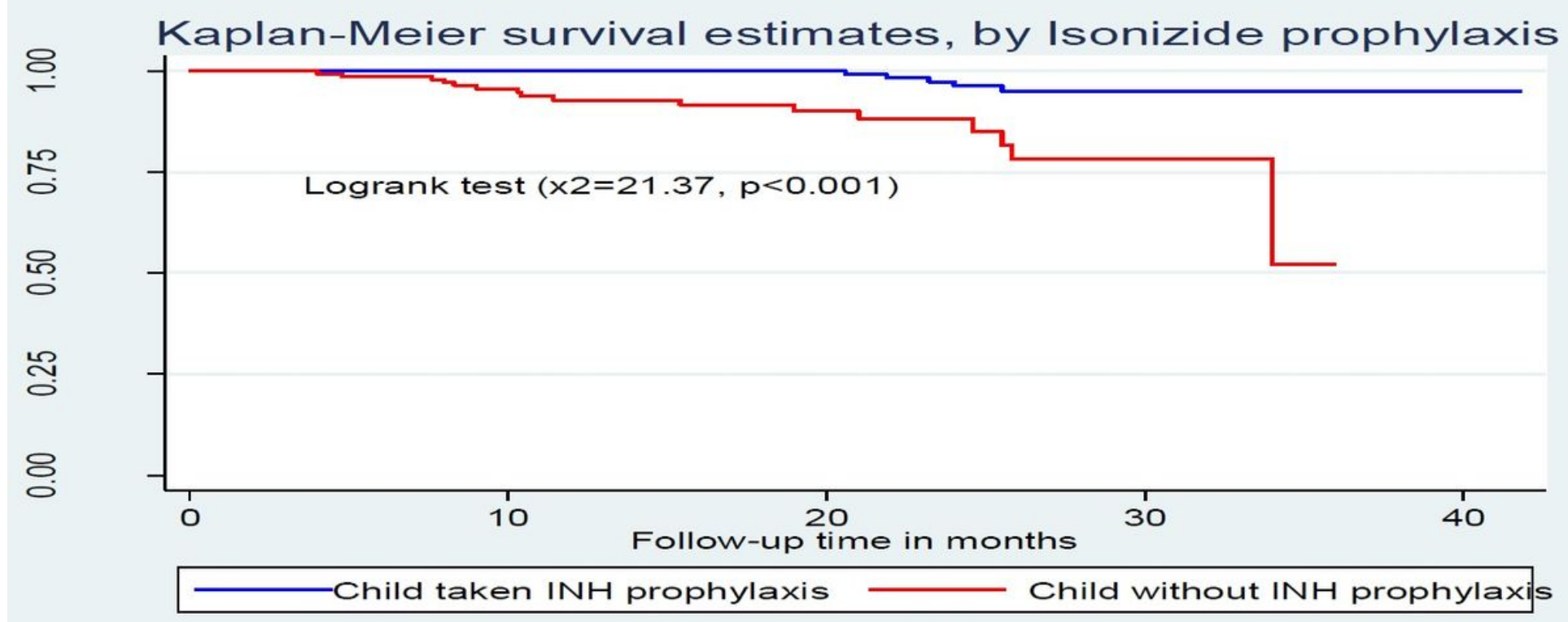

\section{Figure 5}

Kaplan-Meier survival estimates based on their Isoniazide prophylaxis among under five children on ART in Oromia 'liyu' Zone, Amhara region, Ethiopia 2020. 